\title{
INDICADORES DE CONFORT RELACIONADOS CON EL DESEMPEÑO ACADÉMICO DE LOS ESTUDIANTES UNIVERSITARIOS
}

\author{
Luisana C. Bracho Pernalete* \\ https://orcid.org/0000-0001-8428-8985
}

RECIBIDO: Junio 2021 / ACEPTADO: Noviembre 2021 / PUBLICADO: Enero 2022

Como citar: Bracho Pernalete, Luisana. (2022). Indicadores de confort relacionados con el desempeño académico de los estudiantes universitarios. Telos: revista de Estudios Interdisciplinarios en Ciencias Sociales, 24 (1), Venezuela. (Pp. 123-138).

DOI: www.doi.org/10.36390/telos 241.08

\section{RESUMEN}

La presente investigación tiene como finalidad determinar los indicadores del confort que tienen relación con el desempeño académico de los estudiantes universitarios. Para lograr esto, se inició aplicando el cuestionario desarrollado por Gento y Vivas (2003) que constaba de 94 preguntas cerradas bajo una escala Likert compuesta por 5 niveles de satisfacción con respecto a las condiciones de confort de la Institución, distribuidas en 6 dimensiones, a través de un muestreo aleatorio estratificado, aplicado a una muestra conformada por 336 estudiantes de la Facultad de Ingeniería de la Universidad del Zulia, por lo que se puede decir que el presente estudio es de tipo correlacional, transeccional y de campo. Luego de aplicar el cuestionario, se procedió a realizar los análisis estadísticos respectivos, iniciando por las pruebas de normalidad para determinar las pruebas específicas a aplicar para estudiar las correlaciones planteadas por dimensión y para cada uno de los indicadores. Posteriormente se realizaron los análisis respectivos, encontrando finalmente 17 correlaciones significativas de las 94 preguntas de las que constaba el cuestionario, y con éste resultado se pudo construir un diagrama de Ishikawa que describe el comportamiento y las relaciones de las variables en estudio para los estudiantes de la facultad de Ingeniería de La Universidad del Zulia al momento de la medición, notándose que las dimensiones que evidenciaron un mayor número de correlaciones significativas eran las del Proceso de Enseñanza - Aprendizaje y Relaciones Personales.

Palabras Clave: Confort, Desempeño Académico, Satisfacción Estudiantil, Análisis Estadístico, Análisis de Correlación.

\footnotetext{
* Doctora en Ingeniería. Magister Scientiarum en Gerencia de Mantenimiento. Especialista en Ingeniería de Seguridad. Ingeniero Industrial. Docente - Investigadora de la Escuela de Ingeniería Industrial de La Universidad del Zulia. Profesora Asociada. Correo electrónico: luisanabracho@gmail.com
} 


\title{
Comfort indicators related to the academic performance of university students
}

\begin{abstract}
The main objective of this research is to determine which indicators of comfort are related to the academic performance of university students. To achieve this, it was initiated by applying the questionnaire developed by Gento and Vivas (2003) consisting of 94 closed questions under a Likert scale, composed by 5 levels of satisfaction about the comfort conditions of the Institution distributed in 6 dimensions, specifically through stratified random sampling, applied to a sample consisting of 336 students of the Engineering Faculty of the University of Zulia, so it can be said that the present study is correlational, transectional and field type. After applying the questionnaire, the respective statistical analyzes were carried out, starting with the normality tests to determine the specific exams to be applied to study the proposed correlations by dimension and by each one of the indicators in study. Subsequently the respective analyzes were performed, finally finding 17 significant correlations of the 94 questions that were included in the questionnaire, and with this result an Ishikawa diagram was constructed, and it describes the behavior and the relations existing between the variables under study for the students of the faculty of Engineering of the University of Zulia at the moment of the measurements, noting that the dimensions that showed a greater number of significant correlations were the Teaching Learning Process and Personal Relationships.
\end{abstract}

Key Words: Comfort Indicators, Academic Performance, Students Satisfaction, Statistical Analysis, Correlation Analysis.

\section{Introducción}

En los últimos años se han venido incrementando los estudios acerca de las condiciones de confort en las instituciones educativas, debido a la necesidad de conocer de qué manera pueden afectar a la satisfacción de los estudiantes, y más importante aún, al desempeño de los mismos. Tomando en consideración el mercado competitivo en el cual los actuales alumnos deberán integrarse para trabajar en un futuro, se puede inferir que los educadores y las instituciones llevan la responsabilidad de formarlos para hacerlos encajar y resaltar en ese mundo. También se hace importante la optimización de los limitados recursos disponibles en las academias y las actuales condiciones climáticas y de alto consumo de energía, que requieren un cambio en las expectativas de la población en cuanto a las condiciones de confort, que ha venido variando significativamente en las últimas décadas (Chappells y Shove, 2005).

Por su parte, el desempeño académico, también conocido como rendimiento escolar, es un concepto estudiado ampliamente y definido por Jimenez (2000; p. 3), como "el nivel de conocimientos de un alumno demostrado en un área o materia comparado con la norma de edad y nivel académico", y la pregunta que se encuentra inmersa en este concepto es ¿Cuáles son los factores que lo afectan? ¿Qué elementos pueden variar el impacto que tiene cada uno de ellos sobre los alumnos? ¿Existen características de la población estudiada como la edad, el género, nivel socio-económico y hasta la inteligencia de los estudiantes que lo pueden condicionar?

Al respecto, investigaciones como la de Cascón (2000) concluyen que el indicador del nivel educativo adquirido "en prácticamente la totalidad de los países desarrollados y en vías de 
desarrollo, ha sido, sigue y probablemente seguirán siendo las calificaciones escolares" (p. 2) y a su vez, éstas son reflejo de las evaluaciones y/o exámenes donde el alumno debe demostrar sus conocimientos sobre las distintas áreas o materias que el sistema considera necesarias y suficientes para su desarrollo como miembro activo de la sociedad, con lo que infiere que el empleo de las calificaciones obtenidas por los estudiantes debería ser información suficiente para medir de manera cuantitativa el desempeño académico de los mismos.

Sin embargo, en el caso específico de la Universidad del Zulia, se ha venido implementando un sistema para cuantificar el desempeño estudiantil empleado para otorgar el orden de inscripción de los estudiantes, el cual considera además de las calificaciones obtenidas, los indicadores eficiencia y eficacia. La eficacia se calcula en función de la relación entre las materias aprobadas versus materias inscritas. Por su parte, la eficiencia, se refiere a la relación entre el número de materias aprobadas con respecto a las materias disponibles para cada período académico. Para la presente investigación, se considerarán los 3 indicadores para calcular el desempeño académico, ya que la muestra estudiada pertenece a dicha institución.

Como se puede observar, la importancia de la medición de dicha variable radica en que es un indicador que permite determinar el grado en el que la información suministrada a través del proceso de enseñanza-aprendizaje es absorbida por parte de los estudiantes, y a su vez constituye un indicador del logro de los alumnos, sin embargo, este aspecto puede verse afectado por el ambiente de aprendizaje en donde se desenvuelven los estudiantes, por lo que se hace necesario conocer los factores que lo pueden condicionar.

Estos elementos, abarcan desde aspectos físicos hasta aquellos que pueden afectar el bienestar mental de los estudiantes, y pueden ser englobados dentro del concepto de confort, el cual es definido por Kolcaba y Kolcaba (1999), como un "estado de tranquilidad y comodidad" (p. 2), por lo que se puede decir que comprende la sensación de armonía entre los factores que intervienen en el proceso educativo, como plantean algunas investigaciones como la de Pinto et al (2016), quienes también explican que la palabra confort, puede ser usada como un sinónimo de bienestar y de calidad de vida.

Si se toma en consideración el concepto que Surdez (2018) otorga a la satisfacción estudiantil, como "el bienestar que experimentan los alumnos por sentir cubiertas sus expectativas académicas como resultado de las actividades que realiza la institución para atender sus necesidades educativas" (p. 4) se puede inferir que el empleo de un instrumento que mida el nivel de satisfacción de los estudiantes con respecto a dichas condiciones se hace pertinente.

Se hace interesante destacar la amplitud del concepto de confort. En las ramas médicas, se asocia este término al alivio del dolor (Lombardo et al, 2013) o en función de la traducción de la palabra francesa "comfort", la cual significa "sensación de alivio" (Olausson, 2019, p. 10). Desde el punto de vista arquitectónico, se refiere "al conjunto de condiciones ambientales aceptadas por las personas para el desarrollo de sus actividades habituales; las climáticas, pero también del usuario (aspectos fisiológicos, culturales y psicológicos)... y dependen en gran medida de la apreciación de las personas" (D’Alençon, 2008, p. 219).

En las áreas técnicas, se encuentra enmarcada en las características del medioambiente y de la ergonomía, y al respecto, Cole et al (2008) plantean que un estudio convencional de las condiciones de confort analiza las dimensiones clásicamente abordadas, las cuales son: temperatura, iluminación, ruido y calidad del aire, y que son descritas principalmente bajo la 
percepción individual y psicológica de los usuarios, lo cual conduce al último enfoque, relacionado con las ciencias sociales y humanísticas, para la cual Tomiyama et al (2015), plantean que "algunos investigadores la han caracterizado como la reducción de la respuesta biológica al estrés, mientras que otros se enfocan en la reducción de las emociones negativas" (p. 3). En la presente investigación, se emplea una combinación del enfoque técnico y humanístico, al analizar dimensiones relacionadas con las características ambientales, junto con otras de carácter psicosocial.

Muchos estudios se han desarrollado para determinar cuáles son las condiciones ideales para el desarrollo de las actividades académicas, ya que el proceso de aprendizaje puede verse afectado por las características ambientales, como fue demostrado por Alfa et al (2019), quienes encontraron en su estudio en Nigeria que no solo dependía de la experticia del estudiante o sus habilidades de comunicación, sino también de la salubridad y confort del ambiente de aprendizaje proporcionado para tal fin. Del mismo modo, Santos et al (2018), en su investigación desarrollada en Portugal y Brasil demostraron la importancia de mantener los indicadores de confort evaluados (calidad del aire, temperatura, acústica, iluminación y ergonomía) dentro del rango considerado aceptable, específicamente para entornos escolares, ya que los estudiantes permanecen largos períodos dentro de la escuela y las condiciones de confort interfieren en la salud, la concentración y el aprendizaje de los estudiantes.

Entre las investigaciones que se han llevado a cabo en las instituciones educativas, específicamente en las Universidades, en las que se ha estudiado la relación entre el desempeño académico y las condiciones de confort, se han encontrado desde los más específicos, en los que se ha analizado la relación de una sola variable con el desempeño académico, hasta los más amplios que englobaban una gran cantidad de aspectos, sin embargo, para el presente estudio, se emplearán las variables consideradas por Gento y Vivas (2003) e incluidas en el cuestionario diseñado por los autores para conocer la satisfacción de los estudiantes universitarios con su educación. A continuación se explican brevemente los diferentes aspectos que lo engloban.

En el caso de las condiciones básicas de la infraestructura, se ha determinado que el estrés causado por el ambiente de estudio puede menoscabar la habilidad mental y física de los estudiantes, y se ha propuesto, en algunos estudios como el de Yewande y Zafer (2020), que los salones de clase deben ser diseñados empleando los principios del diseño verde, incluyendo además de una buena iluminación, temperatura, y acústica aspectos como forma, orientación, ventanales, entre otros (p. 4).

Otro aspecto a ser considerado es la calidad de los Servicios Estudiantiles, la cual según D'Armas et al (2018), puede ser cuantificada mediante la "demostración de juicios 0 concepciones de la imagen institucional sobre el contexto del sistema educativo" (p. 3). Sin embargo, Gento y Vivas (2003), consideran que esta variable puede ser medida a través del nivel de satisfacción que muestran los alumnos con la manera en que funcionan los mismos, y que "incluyen aspectos tales como las bibliotecas, oficinas de atención estudiantil, comedores, servicios médicos, transporte, entre otros" (p. 6).

Por otro lado, se encuentran algunos aspectos psicosociales relacionados con el confort que deben ser valorados para conocer la manera en la que afectan a los estudiantes, por ejemplo, Darling-Hammond (2000), realizó un estudio extenso sobre la influencia de la calidad de los profesores y sus métodos de enseñanza en el desempeño estudiantil, resultando evidente que 
hay elementos que califican a los profesores como altamente preparados, como certificaciones y títulos en el área en la que imparten clases, y que "estas características son altamente significativas y se correlacionan positivamente con los resultados estudiantiles" (p. 38).

Por su parte, algunas variables como una baja condición económica mostraron una correlación negativa con el desempeño y fue usualmente significativa también. Considerando que la presente investigación es desarrollada en una institución de carácter público, la consideración a las condiciones económicas de los alumnos resulta un aspecto importante a ser medido.

Otro aspecto resaltante a ser evaluado es el relativo a las relaciones personales, y algunos estudios como el de Li et al (2018), han encontrado correlaciones positivas entre la sensación de apoyo social y el autoestima, y a su vez, del autoestima con el desempeño académico ( $p$. 10), lo cual muestra la importancia del estudio de la influencia de la satisfacción de los estudiantes con este elemento. Por su parte, Medina et al (2018), concluyeron que algunos factores personales, como aquellos relacionados con duelos, relaciones de pareja, entre otros pueden afectar el rendimiento académico de los alumnos (p. 23).

Indudablemente, el proceso de Enseñanza-Aprendizaje juega un papel fundamental en la educación de los alumnos, sin embargo, existen numerosos estilos de enseñanza, y a su vez, muchos estilos de aprendizaje, por lo que se hace interesante conocer si estos estilos pueden ser relacionados con el desempeño de los mismos. Al respeto, Yildirim et al (2019), realizaron una extensa revisión bibliográfica, y concluyeron que cualquier técnica de aprendizaje empleada va a conducir a resultados positivos en los estudiantes (p. 19), siempre y cuando sea seleccionada por cada alumno, y que aquellos que se ven más influenciados por las técnicas de enseñanza son los estudiantes de niveles inferiores.

Finalmente se encuentra la modernización de los procesos y de las herramientas empleadas, lo cual constituye un factor importante en la adaptación de los estudiantes a las condiciones y elementos con los que se encontrarán en sus futuros puestos de trabajo. Al respecto, Kriukova y Ameridze (2021), utilizan el término innovación, la cual consideran que existe en varias esferas de la actividad humana, pero en educación, implica el empleo de nuevos conocimientos, técnicas, herramientas, tecnologías, entre otros, para obtener como resultado servicios educativos que satisfagan las demandas sociales y del mercado (p. 15)

El presente estudio pretende proporcionar una mirada desde el punto de vista de los estudiantes de la Facultad de Ingeniería de la Universidad del Zulia acerca de su percepción con respecto a las condiciones de confort de la misma, a través de la medición de su satisfacción con respecto a las dimensiones que pueden afectarla, abarcando no solo las condiciones físicas, sino también el suministro de servicios y algunas variables psicológicas que pueden condicionar la sensación de bienestar y satisfacción de los mismos, para luego determinar, si existe relación entre las condiciones de confort de la institución y el desempeño académico de los estudiantes, analizando finalmente cuáles son las variables que lo condicionan.

\section{Metodología}

Para el desarrollo de la presente investigación, se procedió a aplicar un cuestionario que midiera la satisfacción de los estudiantes con las condiciones de confort de la institución, por lo que se empleó el instrumento SEUE (Satisfacción de los Estudiantes Universitarios con su Educación) diseñado por Gento y Vivas (2003), a una muestra aleatoria calculada para una población finita o de tamaño conocido ( $p=2.653$ alumnos), con un error del $5 \%$ y confiabilidad 
del 95\%, que resultó en 336 estudiantes activos de la Facultad de Ingeniería de la Universidad del Zulia. Conociendo el tamaño de la muestra, se procedió a aplicarla de manera aleatoria, proporcional y estratificada en función de la cantidad de estudiantes activos en cada una de las 7 escuelas que conforman la Facultad de Ingeniería durante el transcurso del primer período académico del año 2018.

A las respuestas obtenidas se le aplicaron las pruebas de normalidad de Kolmogorov Smirnov para conocer las tendencias de los datos y el tratamiento estadístico a emplear para el procesamiento de la información.

En función de estos resultados, fueron analizadas las respuestas obtenidas de cada sujeto, contrastando el puntaje obtenido en cada dimensión y el total con su desempeño académico, el cual fue calculado a través del promedio de sus calificaciones, su eficiencia y su eficacia, otorgándole el mismo peso a cada uno de estos factores.

Finalmente se empleó el índice de correlación de Spearman para determinar las relaciones entre el desempeño académico total con cada uno de los 94 indicadores que medían la satisfacción. Con estos resultados se construyó un diagrama de Ishikawa en el que se podían observar las correlaciones existentes y para poder analizar finalmente los hallazgos obtenidos.

\section{Análisis de los Resultados}

Para iniciar, se procedió a realizar la prueba de Alfa de Cronbach a los resultados obtenidos luego de aplicar la encuesta aleatoriamente al grupo de 336 estudiantes activos de la Facultad de Ingeniería de la Universidad del Zulia. Como se puede observar en la Tabla 1, la Confiabilidad del Instrumento resultó muy Alta, por lo que se decidió que éste instrumento podía ser empleado para la realización del presente estudio.

\section{Tabla 1. Estadísticos de fiabilidad Instrumento}

\begin{tabular}{|r|rr|}
\hline \multicolumn{2}{|c|}{ Alfa de Cronbach } & \multicolumn{2}{|c|}{ N de elementos } \\
\hline &, 964 & \\
\hline
\end{tabular}

Fuente: Elaboración Propia

Luego de asegurar la confiabilidad del instrumento, se procedió a realizar los estudios respectivos a la data recolectada dimensión por dimensión, cuyos indicadores se encuentran distribuidos como se muestra en la Tabla 2:

Tabla 2. Resumen de Resultados Análisis Factorial

\begin{tabular}{|c|c|c|c|}
\hline Dimensión & Nombre & Items que la integran & $\begin{array}{c}\# \\
\text { Items }\end{array}$ \\
\hline Dim. 1 & $\begin{array}{l}\text { Condiciones } \\
\text { Físicas }\end{array}$ & 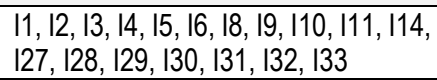 & 18 \\
\hline Dim. 2 & $\begin{array}{l}\text { Servicios } \\
\text { Estudiantiles }\end{array}$ & $\begin{array}{l}17,|12,| 13,|115,| 16,|117,| 18,|21,| 122, \\
I 23,|24,| 25,|46,147,| 162 .\end{array}$ & 15 \\
\hline Dim. 3 & $\begin{array}{l}\text { Accesibilidad } \\
\text { Económica a } \\
\text { Servicios }\end{array}$ & $\begin{array}{l}126,134,135,136,137,138,139,140 \text {, } \\
148 .\end{array}$ & 9 \\
\hline Dim. 4 & $\begin{array}{l}\text { Relaciones } \\
\text { Personales } \\
\end{array}$ & $\begin{array}{l}142,143,144,145,149,150,151,152, \\
153,154,155,156,157,158,184 .\end{array}$ & 15 \\
\hline
\end{tabular}




\begin{tabular}{|c|c|c|c|}
\hline Dim. 5 & $\begin{array}{l}\text { Proceso } \\
\text { Enseñanza - } \\
\text { Aprendizaje }\end{array}$ & $\begin{array}{l}141,159,160,161,164,165,166,167, \\
168,169,173,178,179,180,181,182, \\
183,185,186,187,188,189,190,191, \\
192,193,194 .\end{array}$ & 27 \\
\hline Dim. 6 & $\begin{array}{l}\text { Inserción al } \\
\text { contexto mundial }\end{array}$ & $\begin{array}{l}\text { I19, I20, I63, I70, I71, I72, I74, I75, } \\
176,177 .\end{array}$ & 10 \\
\hline
\end{tabular}

Fuente: Elaboración Propia

Para realizar los análisis respectivos, se procedió a estudiar cada uno de los factores que se iba a correlacionar, ya que el tipo de análisis varía dependiendo del comportamiento de la data, es decir, si sigue una distribución normal o si no lo hace, como se ha demostrado en diversas investigaciones como las desarrolladas por Navarro (2015), Cervantes et al (2004), Rubio y Berlanga (2012) y Castañeda et al (2010), y por lo tanto se siguió la metodología planteada por dichos autores, por lo que se aplicaron las pruebas de bondad de ajuste o normalidad de Kolmogorov Smirnov a cada conjunto de datos recolectados.

En los casos en los que la data se comporte como una distribución normal y se estén cruzando una variable cualitativa con una cuantitativa, se aplican pruebas paramétricas como la $t$ de student o Anova. En el caso de que ambas variables sean cuantitativas se aplica Pearson si las variables son continuas, y si son categóricas se procesa la data como si fuera cualitativa con más de 3 categorías, y se usa Anova.

En aquellos casos en los que no se comporte como una distribución normal se aplican pruebas No Paramétricas; si se están cruzando una variable cualitativa con una cuantitativa se aplica U de Mann Witney o Kruskall Wallis, y si ambas variables son cuantitativas se usa Spearman cuando las variables son continuas, y si son categóricas se procesa la data como si fuera cualitativa con más de 3 categorías, y se emplea por lo tanto Kruskall Wallis.

Luego de haber realizado todas las correlaciones pertinentes, se detectó que casi ninguna de las dimensiones mostraba un nivel de correlación estadísticamente significativo con respecto al desempeño académico, como se puede observar en la tabla 3 que se muestra a continuación:

Tabla 3. Análisis de Correlaciones Dimensiones Vs Desempeño Académico

\begin{tabular}{|c|c|c|c|c|}
\hline \multicolumn{5}{|c|}{ Dimensión 1. Condiciones Físicas } \\
\hline \multirow[t]{2}{*}{ Promedio } & Prueba & Spearman & Sig. Asint. Bilateral & 0,612 \\
\hline & Se acepta $\mathrm{Ho}$ ? & \multicolumn{3}{|c|}{\begin{tabular}{l|l} 
SI & No existe correlación entre las variables \\
\end{tabular}} \\
\hline \multirow[t]{2}{*}{ Eficiencia } & Prueba & Spearman & Sig. Asint. Bilateral & 0,228 \\
\hline & Se acepta $\mathrm{Ho}$ ? & \multicolumn{3}{|c|}{ No existe correlación entre las variables } \\
\hline \multirow[t]{2}{*}{ Eficacia } & Prueba & Spearman & Sig. Asint. Bilateral & 0,103 \\
\hline & Se acepta $\mathrm{Ho}$ ? & \multicolumn{3}{|c|}{ No existe correlación entre las variables } \\
\hline \multirow[t]{2}{*}{ Desempeño } & Prueba & Spearman & Sig. Asint. Bilateral & 0,174 \\
\hline & Se acepta $\mathrm{Ho}$ ? & \multicolumn{3}{|c|}{ No existe correlación entre las variables } \\
\hline \multicolumn{5}{|c|}{ Dimensión 2. Servicios Estudiantiles } \\
\hline \multirow[t]{2}{*}{ Promedio } & Prueba & Spearman & Sig. Asint. Bilateral & 0,941 \\
\hline & Se acepta $\mathrm{Ho}$ ? & \multicolumn{3}{|c|}{ No existe correlación entre las variables } \\
\hline \multirow[t]{2}{*}{ Eficiencia } & Prueba & Spearman & Sig. Asint. Bilateral & 0,617 \\
\hline & Se acepta $\mathrm{Ho}$ ? & \multicolumn{3}{|c|}{ lación entre las variables } \\
\hline
\end{tabular}


Indicadores de confort relacionados con el desempeño académico de los estudiantes universitarios

\begin{tabular}{|c|c|c|c|c|}
\hline \multirow[t]{2}{*}{ Eficacia } & Prueba & Spearman & Sig. Asint. Bilateral & 0,616 \\
\hline & Se acepta Ho? & \multicolumn{3}{|c|}{ No existe correlación entre las variables } \\
\hline \multirow[t]{2}{*}{ Desempeño } & Prueba & Spearman & Sig. Asint. Bilateral & 0,862 \\
\hline & Se acepta Ho? & \multicolumn{3}{|c|}{ No existe correlación entre las variables } \\
\hline \multicolumn{5}{|c|}{ Dimensión 3. Accesibilidad Económica a Servicios } \\
\hline \multirow[t]{2}{*}{ Promedio } & Prueba & Spearman & Sig. Asint. Bilateral & 0,388 \\
\hline & Se acepta Ho? & \multicolumn{3}{|c|}{ No existe correlación entre las variables } \\
\hline \multirow[t]{2}{*}{ Eficiencia } & Prueba & Spearman & Sig. Asint. Bilateral & 0,462 \\
\hline & Se acepta Ho? & \multicolumn{3}{|c|}{ No existe correlación entre las variables } \\
\hline \multirow[t]{2}{*}{ Eficacia } & Prueba & Spearman & Sig. Asint. Bilateral & 0,620 \\
\hline & Se acepta Ho? & \multicolumn{3}{|c|}{ No existe correlación entre las variables } \\
\hline \multirow[t]{2}{*}{ Desempeño } & Prueba & Spearman & Sig. Asint. Bilateral & 0,417 \\
\hline & Se acepta Ho? & \multicolumn{3}{|c|}{ No existe correlación entre las variables } \\
\hline \multicolumn{5}{|c|}{ Dimensión 4. Relaciones Personales } \\
\hline \multirow[t]{2}{*}{ Promedio } & Prueba & Spearman & Sig. Asint. Bilateral & 0,437 \\
\hline & Se acepta Ho? & \multicolumn{3}{|c|}{ No existe correlación entre las variables } \\
\hline \multirow[t]{2}{*}{ Eficiencia } & Prueba & Spearman & Sig. Asint. Bilateral & 0,067 \\
\hline & Se acepta Ho? & \multicolumn{3}{|c|}{ No existe correlación entre las variables } \\
\hline \multirow[t]{2}{*}{ Eficacia } & Prueba & Spearman & Sig. Asint. Bilateral & 0,164 \\
\hline & Se acepta Ho? & No exi & correlación entre las & \\
\hline Desempeño & Prueba & Spearman & Sig. Asint. Bilateral & 0,098 \\
\hline & Se acepta Ho? & No exis & correlación entre las & \\
\hline & Dimensión & eso Enseñan & - Aprendizaje & \\
\hline Promedio & Prueba & Spearman & Sig. Asint. Bilateral & 0,288 \\
\hline & Se acepta Ho? & \begin{tabular}{l|l} 
SI & No exis \\
\end{tabular} & correlación entre las & \\
\hline Eficiencia & Prueba & Spearman & Sig. Asint. Bilateral & 0,009 \\
\hline & Se acepta Ho? & Si ex & e correlación entre las & \\
\hline Eficacia & Prueba & Spearman & Sig. Asint. Bilateral & 0,064 \\
\hline & Se acepta Ho? & No ex & e correlación entre las & \\
\hline Desempeño & Prueba & Spearman & Sig. Asint. Bilateral & 0,025 \\
\hline & Se acepta Ho? & Si ex & e correlación entre las & \\
\hline & Dimensi & serción al col & exto mundial & \\
\hline Promedio & Prueba & Spearman & Sig. Asint. Bilateral & 0,065 \\
\hline & Se acepta $\mathrm{Ho}$ ? & No e & te correlación entre la & \\
\hline Eficiencia & Prueba & Spearman & Sig. Asint. Bilateral & 0,236 \\
\hline & Se acepta Ho? & No e & ste correlación entre la & \\
\hline Eficacia & Prueba & Spearman & Sig. Asint. Bilateral & 0,035 \\
\hline & Se acepta Ho? & Si ex & e correlación entre las & \\
\hline Desempeño & Prueba & Spearman & Sig. Asint. Bilateral & 0,072 \\
\hline & Se acepta Ho? & No e & te correlación entre la & \\
\hline
\end{tabular}

Fuente: Elaboración Propia 
Como se puede observar, fueron muy pocas las correlaciones significativas halladas entre las seis dimensiones medidas con el desempeño académico, y con los 3 factores que lo integran (promedio, eficiencia y eficacia), por lo que se puede decir que el modelo no se ajusta adecuadamente a la hipótesis planteada, ya que solo se ajusta en el caso de la Eficiencia con las dimensiones 5 y 6 . Por su parte, se hace importante resaltar que la Dimensión 5 es la única que muestra un nivel de correlación estadísticamente significativo con el desempeño, lo cual es importante, ya que ésta dimensión se refiere al Proceso de Enseñanza - Aprendizaje, y era esperable que mostrara algún tipo de reciprocidad.

También se determinó que la Eficiencia (materias aprobadas / materias sugeridas) mostraba correlación con la misma dimensión y con la dimensión 6 (Inserción al Contexto Mundial), lo cual se hace interesante, porque demuestra la importancia que le dan los estudiantes a los estándares planteados por la Universidad y al mercado laboral nacional y mundial, tratando de aprovechar al máximo los recursos disponibles.

Evaluación ítem por ítem

Como las dimensiones no mostraron tener mucha relación, se procedió a realizar unas pruebas de hipótesis con cada uno de los ítems que conforman las dimensiones con respecto al desempeño académico total. Como la muestra no presenta una distribución Normal, de acuerdo a los resultados obtenidos al aplicar las pruebas de Kolmogorov Smirnov, se realizaron los análisis a través del cálculo del Rho de Spearman, y los resultados significativos de las correlaciones para cada una de las dimensiones se muestran en la Tabla 4 presentada a continuación.

Tabla 4. Correlaciones Significativas Item por Item Vs Desempeño Académico

\begin{tabular}{|c|c|c|c|c|c|c|c|c|}
\hline & $\operatorname{Dim} 1$ & \multicolumn{2}{|c|}{ Dimensión 2} & Dim. 3 & \multicolumn{2}{|c|}{ Dim. 4} \\
\hline & & & 105 & 107 & 123 & 136 & 142 & 143 \\
\hline Rho de & DESEMPEÑO & Coef. De Corr &,- 112 &,- 141 &, 113 & ,120 & ,278 & ,229 \\
\hline Spearman & & Sig. (bilateral) & ,040 & ,010 & ,038 & ,028 & ,000 & ,000 \\
\hline & & $\mathrm{N}$ & 336 & 336 & 336 & 336 & 336 & 336 \\
\hline
\end{tabular}

\begin{tabular}{|c|c|c|c|c|c|c|c|c|}
\hline & \multicolumn{4}{|c|}{ Dimensión 4} & \multicolumn{2}{|c|}{ Dim. 5} \\
\hline & & & 150 & 151 & 154 & 156 & 161 & 178 \\
\hline Rho de & DESEMPEÑO & Coef. De Corr & 128 & 173 & 155 & 110 & 119 & 268 \\
\hline Spearman & & Sig. (bilateral) & ,019 & ,002 & ,004 & ,044 & ,029 & ,000 \\
\hline & & $\mathrm{N}$ & 336 & 336 & 336 & 336 & 336 & 336 \\
\hline
\end{tabular}

\begin{tabular}{|l|l|l|r|r|r|r|r|}
\hline \multicolumn{2}{|c|}{} & \multicolumn{5}{|c|}{ Dimensión 5 } & Dim.6 \\
\hline \multicolumn{2}{|l|}{} & 180 & 182 & 185 & 186 & 176 \\
\hline Rho de & DESEMPEÑO & Coef. De Corr &, 250 &, 113 &, 127 &, 190 &,- 196 \\
Spearman & & Sig. (bilateral) &, 000 &, 038 &, 020 &, 000 &, 000 \\
& & $\mathrm{~N}$ & 336 & 336 & 336 & 336 & 336 \\
\hline
\end{tabular}

Fuente: Elaboración Propia

Se puede observar que solo uno de los Indicadores medidos a través de la 1era Dimensión (Condiciones Físicas), y es el que se refiere a la Comodidad del Mobiliario, se correlacionó con el desempeño académico de los estudiantes.

En cuanto a la segunda dimensión, se puede notar que existen dos correlaciones altamente significativas, pertenecientes a los Items 07 y 23 , los cuales corresponden a los espacios disponibles para el estudio y la cantidad de alimentos ofrecidos en el comedor 
estudiantil respectivamente. El primero se refiere a la existencia de lugares en los cuales los alumnos puedan estudiar o practicar, y el segundo trata de la importancia que tiene para los alumnos la disponibilidad de un comedor que los ayude a poder alimentarse adecuadamente al menos una vez al día, y en el caso de los estudiantes foráneos, dos veces al día.

Adicionalmente, se puede observar que solo hay una correlación significativa de los Indicadores medidos a través de la 3era Dimensión (Accesibilidad económica a servicios), y es el referido al servicio del comedor, lo cual es muy interesante, ya que es la segunda vez que una variable relacionada al comedor estudiantil sale a relucir, aspecto que llama poderosamente la atención y lleva a pensar que una de las motivaciones de los estudiantes para asistir a clases es la posibilidad de consumir el almuerzo de manera gratuita. Se hace importante también destacar la importancia de una alimentación balanceada en los estudiantes; estudios desarrollados en otros países, como el de Raskind et al (2018), en Estados Unidos, concluyeron que aquellos estudiantes universitarios que han experimentado dificultades para acceder a una alimentación constante y balanceada han enfrentado obstáculos en su desempeño académico, y hasta en su salud psicosocial (p. 8), y esto constituye un problema que debe ser atendido.

En la cuarta dimensión, referida a las Relaciones Interpersonales de los estudiantes, se nota un mayor número de correlaciones que en los casos anteriores, evidenciándose las relaciones con los Items:

- 142. Sus compañeros de grupo

- 143. Los otros alumnos de la universidad

- 150. Los jefes de departamentos

- 151. Los profesores

- $\quad$ 154. Los compañeros de clase

- $\quad$ 156. Consideración y respeto a sus ideas y propuestas en el aula

Como se puede observar, el desempeño de los estudiantes se ve altamente correlacionado por el trato recibido por aquellas personas con quienes interactúan a diario, conformado por sus amigos, compañeros de clase y profesores (los jefes de departamento de cada escuela son profesores de la misma), y también el respeto mostrado ante sus opiniones.

En la quinta dimensión, que se refiere a la percepción del proceso de Enseñanza Aprendizaje, es donde se observa el mayor número de correlaciones significativas, presentes en los ítems 169, 178, 180, 182, 185 e 186, los cuales se refieren a:

- $\quad$ 169. El régimen de estudios (anual/semestral).

- 178. Los resultados o calificaciones obtenidas.

- $\quad$ 180. El dominio de técnicas de estudio y trabajo intelectual alcanzadas.

- $\quad$ 82. Las habilidades y destrezas para el trabajo docente desarrolladas.

- $\quad$ 185. Reconocimiento del éxito personal por parte de los profesores.

- $\quad$ 186. Reconocimiento del éxito personal por parte de los alumnos de su grupo.

Se hace interesante observar los indicadores del proceso de enseñanza - aprendizaje que más se relacionan con el desempeño estudiantil; se puede notar que tanto las técnicas de enseñanza de los profesores, como las de estudio aplicadas por los estudiantes afectan directamente el desempeño, y también la aspiración a obtener buenas calificaciones para alcanzar el reconocimiento de sus compañeros y profesores. 
Finalmente, en la 6ta dimensión, que se refiere a la Inserción al contexto mundial, sólo se encontró que un ítem era estadísticamente significativo con respecto al desempeño académico, y era el I76, el cual mide la Incorporación de las nuevas tecnologías a la enseñanza, y se refiere al empleo de técnicas modernas de enseñanza que permitan reforzar el conocimiento adquirido por parte de los estudiantes en clase.

Diagrama de Ishikawa

Para observar de manera global los resultados presentados con anterioridad, se procedió a realizar un diagrama de Ishikawa para mostrar las relaciones encontradas, el cual se puede observar en la Figura 1.

Como se ha expresado con anterioridad, en las dimensiones 4 (Relaciones Personales) y 5 (Proceso de Enseñanza - Aprendizaje) es en las cuales se observa un mayor número de correlaciones significativas, por lo que se considera que son los indicadores a los cuales se debe prestar mayor atención al momento de implementar mejoras, ya que son éstos ítems los que podrían modificar positivamente el desempeño académico.

Figura 1. Diagrama de Ishikawa Satisfacción Vs. Desempeño Académico.

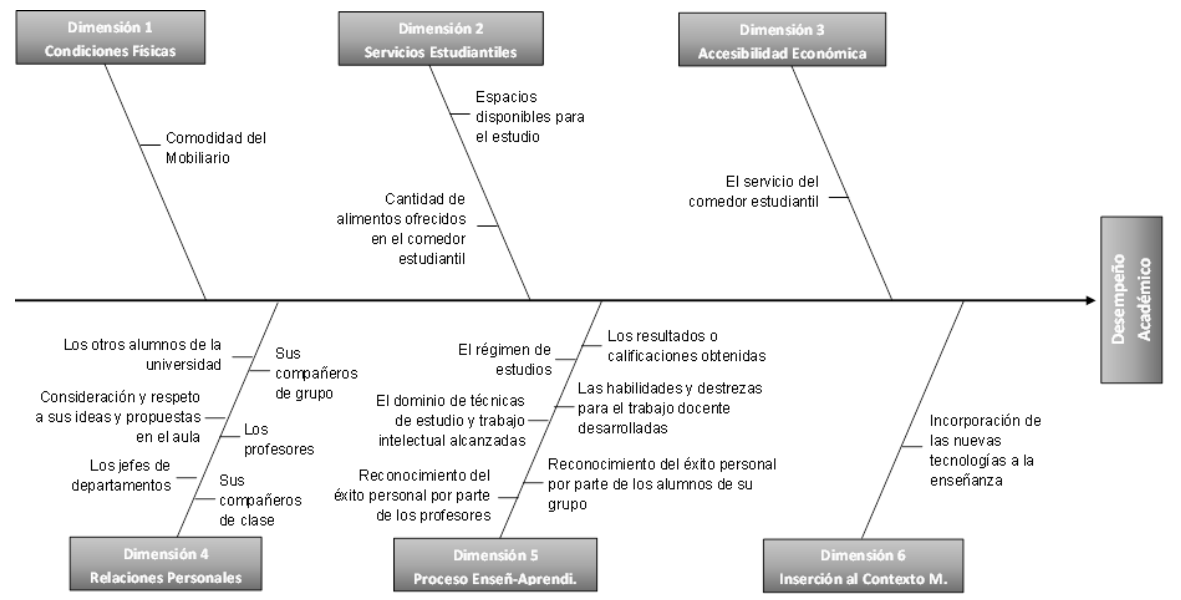

Fuente: Elaboración Propia

Posteriormente, se procedió a analizar estos ítems individualmente para conocer la percepción general de los estudiantes ante cada uno de ellos, en función del valor otorgado al momento de responder el constructo, y adicionalmente, para saber cuáles son los indicadores en condiciones más críticas. Para totalizar, se procedió a organizar estos resultados de acuerdo al valor promedio, y así se obtuvo la Tabla 5:

Tabla 5. Criticidad de los Indicadores Altamente Significativos con el Desempeño

\begin{tabular}{|l|l|l|c|}
\hline Rango & Item & Planteamiento & $\begin{array}{c}\text { Respuesta } \\
\text { Promedio }\end{array}$ \\
\hline \multirow{2}{*}{ Insatisfecho } & 123 & Cantidad de alimentos ofrecidos en el comedor & 2,57 \\
\cline { 2 - 4 } & 107 & Espacios disponibles para el estudio & 2,63 \\
\hline
\end{tabular}


Indicadores de confort relacionados con el desempeño académico de los estudiantes universitarios

\begin{tabular}{|l|l|l|c|}
\hline \multirow{4}{*}{} & 105 & Comodidad del mobiliario & 2,7 \\
\cline { 2 - 4 } & 176 & Incorporación de las nuevas tecnologías a la enseñanza & 2,74 \\
\cline { 2 - 4 } & 136 & El servicio de comedor & 2,81 \\
\hline \multirow{4}{*}{$\begin{array}{c}\text { Poco } \\
\text { Satisfecho }\end{array}$} & 161 & El régimen de estudios (anual/semestral) & 3,26 \\
\cline { 2 - 4 } & 150 & Los jefes de departamento & 3,39 \\
\cline { 2 - 4 } & 178 & Con los resultados o calificaciones obtenidas & 3,51 \\
\cline { 2 - 4 } & 156 & Consideración y respeto a sus ideas y propuestas en el aula & 3,6 \\
\cline { 2 - 4 } & 154 & Los compañeros de clase & 3,75 \\
\cline { 2 - 4 } & 185 & Los otros alumnos de la universidad & 3,77 \\
\cline { 2 - 4 } & 182 & Trato recibido de sus profesores & 3,81 \\
\cline { 2 - 4 } & 186 & Los profesores & 3,88 \\
\cline { 2 - 4 } & 180 & $\begin{array}{l}\text { Con las habilidades y destrezas para el trabajo docente } \\
\text { desarrolladas }\end{array}$ & 3,93 \\
\hline Satisfecho & 142 & Los alumnos de su grupo & 3,96 \\
\hline
\end{tabular}

Fuente: Elaboración Propia

Como se puede observar, algunos de los ítems que resultaron significativos son factores ajenos a la universidad que no pueden ser manejados por ellos, como la satisfacción con sus compañeros de clase, pero afortunadamente se encuentran satisfechos con estos elementos, sin embargo, se observaron varios aspectos interesantes.

Los estudiantes se encuentran insatisfechos con la cantidad de alimentos recibidos en el comedor estudiantil, mostrándose que es el elemento con menor calificación de todos, y en el mismo rango se encuentra el servicio del comedor, es decir, tampoco se sienten cómodos con el trato que reciben dentro del recinto. Adicionalmente, se evidenció que los alumnos perciben que no existen suficientes áreas en las cuales ellos puedan permanecer estudiando fuera de las horas de clase, o que las mismas no poseen condiciones adecuadas para mantenerse dentro de la institución. También les gustaría que el mobiliario fuera más cómodo, debido a las largas jornadas en las que a veces deben permanecer dentro del salón de clases ubicándose en un mismo escritorio o pupitre.

Finalmente, dentro de los aspectos más críticos detectados, se encuentra la incorporación de nuevas tecnologías a la enseñanza, donde los estudiantes se podrían sentir más satisfechos si contaran con algunos elementos tecnológicos que agilicen las clases y que en algunos casos las puedan hacer más dinámicas y efectivas.

Los otros aspectos detectados poseen valores bastante homogéneos y se basan en muchos casos en la opinión percibida acerca del trato recibido por parte del resto de los actores que hacen vida dentro de la institución, notándose igualmente que los aspectos que mostraron una mejor evaluación desde el punto de vista de los estudiantes se basan en las técnicas de enseñanza aplicadas por los profesores y por el dominio de técnicas de estudio por parte de ellos mismos.

\section{Conclusiones}


No se observó correlación entre el nivel de satisfacción total de los estudiantes con respecto a las condiciones de confort y el desempeño académico, por lo que se puede decir que el nivel de satisfacción de los estudiantes muestreados no se encuentra relacionado con su desempeño, y que el mismo podría venir condicionado por algunas variables, tales como las asociadas al proceso de enseñanza-aprendizaje, al ser la única dimensión que mostró un nivel de correlación estadísticamente significativo, y que sería interesante poder estudiar en profundidad en el futuro.

En investigaciones similares, como la de Yildirim et al (2019), se encontraron correlaciones análogas a la del presente estudio con respecto a esta dimensión, sin embargo, al haber desarrollado con mayor amplitud su estudio, encontraron que en planteles de educación básica se nota más la influencia del proceso de Enseñanza - Aprendizaje sobre el desempeño académico. Tomando en consideración que la presente investigación fue desarrollada exclusivamente a nivel universitario y mostró también una correlación significativa, se podría inferir que las características de la población en estudio pueden llevar a resultados diferentes, y que los mismos no pueden ser transferidos.

Se determinó también que la opinión promedio de los estudiantes con respecto a los indicadores que se correlacionaban con el desempeño académico oscilaba entre 2 y 4 puntos, notándose que los ítems con menor valoración se encontraban relacionados con el Comedor Estudiantil y los de mayor nivel de satisfacción con sus compañeros de grupo, su autorrealización y la calidad de la enseñanza recibida por parte de sus profesores, notándose entonces que su sentido de pertenencia al grupo y sus buenas relaciones con sus pares y superiores son aspectos motivadores de los estudiantes. Por otro lado, se debe prestar especial atención en aquellos peores valorados para tratar de desarrollar las mejoras pertinentes en aras de incrementar el desempeño de los estudiantes.

Se hace interesante desarrollar un estudio profundo sobre las condiciones del Comedor Estudiantil, ya que es un factor que mostró correlación con el desempeño de los estudiantes y es el peor valorado por los mismos, por lo que se exhorta a la revisión de las fallas que poseen en el servicio para tratar de mejorar el desempeño académico. Considerando que La Universidad del Zulia es una institución de carácter público, es posible que gran parte de la población estudiantil dependa del servicio de comedores para poder alimentarse adecuadamente y de allí la importancia de la atención a este problema detectado que puede acarrear a dificultades mayores como se ha demostrado en estudios previos como el de Raskind et al (2018) quien concluye que puede afectar hasta en su salud psicosocial.

Las condiciones de los espacios destinados para la permanencia de los estudiantes fuera de las aulas de clase, según la opinión del grupo en estudio son deficientes, por lo que sería pertinente mejorarlas, para lograr que los mismos puedan permanecer en sus instalaciones mientras estudian, esperan sus próximas clases o se reúnen con sus compañeros.

Se propone realizar un estudio para modificar el diseño del mobiliario empleado en los salones de clase considerando las características antropométricas de los estudiantes, ya que los mismos se muestran insatisfechos con respecto a la comodidad de los mesones, sillas y pupitres y ellos deben permanecer largas jornadas bajo estas condiciones. Se sugiere emplear un nuevo diseño como el propuesto por Lam et al (2018), para que sea cómodo, proporcione seguridad y salud a los usuarios, facilite la interacción entre alumnos y profesores y que a su vez sea compatible con el empleo de tecnologías de información, ya que el diseño interior debe crear un ambiente confortable que motive al aprendizaje. 
Finalmente se encontró que otra variable que mostró correlación, es la relacionada con la incorporación de equipos tecnológicos en las aulas que permitan la optimización de las clases y la actualización del contenido impartido en las mismas, por lo que se sugiere desarrollar un plan de inversión que permita la adaptación del proceso de enseñanza - aprendizaje a las condiciones sugeridas por los estudiantes para obtener una mayor motivación en ellos y mejorar así su desempeño académico.

\section{Referencias Bibliográficas}

Alfa, Mohammed; Tauheed, Samuel; Medayese, Obasanjo y Adeola, Owoyale (2019). "Space configuration and learning comfort; a case study of Federal University of Technology Minna lecture halls." Contemporary Educational Researches Journal 9 (1). Chipre. (Pp. 20-31). https://doi.org/10.18844/ceri.v9i1.4058

Cascón, Inocencio. (2000). Análisis de las calificaciones escolares como criterio de rendimiento académico. El Salvador. Extraído de: https://campus.usal.es/ inico/investigacion/jornadas/jornada2/comun/c17.html.

Castañeda, María; Cabrera, Alberto; Navarro, Yadira y de Vries, Wietze. (2010). Procesamiento de datos y análisis estadísticos utilizando SPSS. Un libro práctico para investigadores y administradores educativos. Editorial ediPUCRS. Brasil. Extraido de: http://repositorio.minedu.gob.pe/handle/20.500.12799/4538

Cervantes, Armando; Rivera, Patricia y De la Paz, Juana. (2004) Estadística Práctica para el análisis de datos. Academia de Estadística y Computo Científico. Universidad Nacional Autónoma de México. Facultad de Estudios Superiores. México. Extraído de: https://www.zaragoza.unam.mx/wpcontent/Portal2015/Licenciaturas/biologia/ecocuan/ecocuan_SPSS.pdf.

Chappells, Heather y Shove, Elizabeth (2005) Debating the future of comfort: environmental sustainability, energy consumption and the indoor environment. Building Research \& Information, $33 \quad$ (1). United Kingdom. (Pp. 32-40). https://doi.org/10.1080/0961321042000322762

Cole, Raymond; Robinson, John; Brown, Zosia y O'shea, Meg (2008) Re-contextualizing the notion of comfort, Building Research \& Information, 36 (4). United Kingdom. (pp. 323336), doi: https://doi.org/10.1080/09613210802076328

D'Alençon, Renato. (2008). Acondicionamiento, arquitectura y técnica. Ediciones ARQ. Chile. Extraído de: http://www.edicionesarq.cl/2008/acondicionamientos/

Darling-Hammond, Linda (2000). Teacher Quality and Student Achievement: A Review of State Policy Evidence. Center for the study of teaching and policy, University of Washington. s/v. USA. (Pp. 1 - 48). https://doi.org/10.14507/epaa.v8n1.2000

D'Armas, Mayra; Robles, Rodolfo; Cedillo, Miguel; Vinueza, Jorge; Correa, Mirella; Mejias, Agustin y Acosta, Gina (2018). Imagen institucional universitaria: un estudio de caso en el contexto del Ecuador. Espacios, 39(05). Venezuela (Pp.14-30). Extraído de: http://www.revistaespacios.com/a18v39n05/18390514.html

Gento Palacios, Samuel y Vivas García, Mireya (2003) El SEUE: un instrumento para conocer la satisfacción de los estudiantes universitarios con su educación. Acción Pedagógica. 12 (2). Venezuela. (Pp. 16 - 27). Extraído de: https://dialnet.unirioja.es/servlet/articulo?codigo $=2972060$ 
Jiménez, Manuel. (2000). Competencia social: intervención preventiva en la escuela. Infancia y Sociedad. 24. España.(Pp. 21- 48). Extraído de: https://dialnet.unirioja.es/servlet/articulo?codigo $=4353980$

Kolcaba, Katharine y Kolcaba, Raymond (1991). An analysis of the concept of comfort. Journal of advanced nursing. 16 (11) Estados Unidos. (Pp. 1301-1310). https://doi.org/10.1111/j.1365-2648.1991.tb01558.x

Kriukova, Y. S. y Ameridze, O. S. (2021) Key challenges in introducing innovative technologies into the educational process. XX Congreso Científico y Técnico Internacional "Fabricación de instrumentos: Estado y perspectivas. Kiev, Ucrania. Extraído de: https://ela.kpi.ua/bitstream/123456789/42714/1/PSP-XX_2021_p143-144.pdf

Lam, Edmond; Wong, Irene y Chan, Daniel. (2018) Impacts of Space and Furniture Design on Student Learning. Conference On Striving for Quality Education - FSTE. Hong Kong, China. Extraído de: https://www.fste.edu.hk/conference/2018/files/201822.pdf

Li, Jie; Han, Xue; Wang, Wangshuai; Sun, Gong y Cheng, Zhiming. (2018). How Social Support Influences University Students' Academic Achievement and Emotional Exhaustion: The Mediating Role of Self-Esteem. Learning and Individual Differences. 61. Estados Unidos. (Pp. 120 - 136). https://doi.org/10.1016/j.lindif.2017.11.016

Lombardo, Veronique; Vinatier, Isabelle; Baillot, Marie-Loue; Franja, Vicenta; Bourgeon-Ghittori, Irma; Dray, Sandrine; Jeune, Sylvie; Mossadegh, Chirine; Reignier, Jean; Souweine, Bertrand y Roch, Antoine. (2013) How caregivers view patient comfort and what they do to improve it: a French survey. Annals of Intensive Care. 3(1). Francia. (Pp. 1 - 8). Doi: https://doi.org/10.1186/2110-5820-3-19

Medina, Niorka, Fereira, Johan, \& Marzol, Robinson. (2018). Factores personales que inciden en el bajo rendimiento académico de los estudiantes de geometría. Telos: Revista de Estudios Interdisciplinarios en Ciencias Sociales. 20(1), Venezuela. (Pp. 4-28). https://doi.org/10.36390/telos201.02

Navarro, Enrique (2015) Guía para la interpretación de resultados en el contraste de hipótesis estadísticas. Eduktive. (Versión 5). España. Recuperado en: https://es.slideshare.net/navarroenrique/gua-contraste-de-hiptesis-blog

Olausson, Sepideh, Fridh, Isabell, Lindahl, Berit, \& Torkildsby, Anne-Britt. (2019). The meaning of comfort in the intensive care unit. Critical care nursing, 42(3), Iran. (Pp. 329-341). doi: https://doi.org/10.1097/CNQ.0000000000000268.

Pinto, Sara; Fumincelli, Lais; Mazzo, Alessandra; Caldeira, Silvia y Martins, Jose (2017). Comfort, well-being and quality of life: Discussion of the differences and similarities among the concepts. Porto Biomedical Journal, 2(1), Portugal (Pp. 6-12). https://doi.org/10.1016/i.pbj.2016.11.003

Raskind, llana; Haardörfer, Regine y Berg, Carla. (2019). Food insecurity, psychosocial health and academic performance among college and university students in Georgia, USA. Public Health Nutrition. 22 (3), Estados Unidos. (Pp. 476-485). https://doi.org/10.1017/S1368980018003439

Rubio, Maria Jose. \& Berlanga, Vanesa. (2012) Cómo aplicar las pruebas paramétricas bivariadas t de Student y ANOVA en SPSS. Caso práctico. REIRE. Revista d'Innovació I Recerca en Educació. 5. (2). España (Pp. 83-100). Recuperado en: https://core.ac.uk/download/pdf/41554689.pdf 
Santos, Tatiana; de Almeida, Manuela; Bragança, Luís y Barbosa, Maria Teresa. (2018). Environmental Comfort Indicators for School Buildings in Sustainability Assessment Tools. Sustainability, 10 (6). Suiza. (pp. 1 - 11) -. https://doi.org/10.3390/su10061849 Surdez, Edith; Sandoval, Maria y Lamoyi, Clara. (2018). Satisfacción estudiantil en la valoración de la calidad educativa universitaria. Educación y Educadores, 21(1) Colombia (Pp. 926). https://doi.org/10.5294/edu.2018.21.1.1

Tomiyama, Janeth; Finch, Laura y Cummings, Jenna. (2015). Did that brownie do its job? Stress, eating, and the biobehavioral effects of comfort food. Emerging trends in the social and behavioral sciences, 1 (1) Estados Unidos (Pp. 1-15). Doi: https://doi.org/10.1002/9781118900772.etrds0324.

Yewande, Salimar y Zafer, Halil, (2020) The Construct of Comfort: A Case Study of Environmental Comfort of University Classrooms. United International Journal for Research \& Technology (UIJRT). 1 (06). India (Pp. 19-23). Recuperado en: https://uijirt.com/articles/v1/i6/UIJRTV1/60003.pdf

Yildirim, İbrahim; Cırak-kurt, Sevilay y Sen, Sedat. (2019). The Effect of Teaching Learning Strategies on Academic Achievement: A Meta-Analysis Study. Eurasian Journal of Educational Research, 19 (79). Reino Unido. (Pp. 87-114). Extraído de https://dergipark.org.tr/en/pub/ejer/issue/42986/520736 